\title{
Coherent Noise Reduction in High Visibility Phase-Sensitive Optical Time Domain Reflectometer for Distributed Sensing of Ultrasonic Waves
}

\author{
Hugo F. Martins, Sonia Martin-Lopez, Pedro Corredera, Member, OSA, Massimo L. Filograno, Orlando Frazão, \\ and Miguel González-Herráez, Senior Member, OSA
}

\begin{abstract}
Phase-sensitive optical time domain reflectometry ( $\phi$ OTDR) is a simple and effective tool allowing the distributed monitoring of vibrations along single-mode fibers. Up to now, $\phi$ OTDRs have been used mostly for the measurement of sub-kHz vibrations, normally in the context of intrusion sensing. In this paper, the authors present an experimental and theoretical description of a high-visibility $\phi \mathrm{OTDR}$ and its performance when used for ultrasonic vibration measurements. The use of a semiconductor optical amplifier in the setup allows to suppress coherent noise and also to improve the spectral response of the pump pulses. These two advantages greatly decrease the detected intra-band noise thus allowing frequency measurements in the limits set by the time of flight of the light pulses while maintaining the simplicity of the scheme, as no post-processing, extremely high coherence lasers or coherent detection methods are required. The sensor was able to measure vibrations of up to $39.5 \mathrm{kHz}$ with a resolution of $5 \mathrm{~m}$ over a range which could go up to $1.25 \mathrm{~km}$. This is the first time to our knowledge that a fully distributed measurement of ultrasonic waves was achieved. The statistical behavior of the system was also described theoretically and characterized experimentally.
\end{abstract}

Index Terms-Distributed sensor, optical fiber sensors, phasesensitive OTDR, vibration measurements.

\section{INTRODUCTION}

$\mathbf{F}$ IBER optic distributed sensing technology offers clear advantages over conventional point sensors when the number

Manuscript received June 25, 2013; revised September 10, 2013; accepted October 14, 2013. Date of publication October 17, 2013; date of current version November 6, 2013. This work was supported in part by funding from the European Research Council through Starting Grant U-FINE (Grant no. 307441), in part by the Spanish "Plan Nacional de I+D+i" through projects TEC201237958-C02-01 and TEC2012-37958-C02-02, and in part by the regional program FACTOTEM2 funded by the Comunidad de Madrid and the INTERREG SUDOE program ECOAL-MGT. HFM acknowledges funding from the Portuguese Fundação para a Ciência e Tecnologia (FCT) for providing his $\mathrm{PhD}$ Scholarship, SFRH/BD/76991/2011.

H. F. Martins is with the Faculdade de Ciências da Universidade do Porto, Rua do Campo Alegre, 687, 4169007 Porto, Portugal and also with INESC TEC, Rua do Campo Alegre, 687, 4169007 Porto, Portugal (e-mail: hfm@inescporto.pt).

S. Martins-Lopez and M. González-Herráez are with the Departamento de Electrónica, Universidad de Alcalá, Escuela Politécnica, 28871 Madrid, Spain (e-mail: sonia.martin@depeca.uah.es; miguel.gonzalezh@uah.es).

P. Corredera and M. L. Filograno are with the Instituto de Óptica, CSIC. C/Serrano 144, 28006 Madrid, Spain (e-mail: p.corredera@csic.es; m.f@csic.es).

O. Frazão is with the INESC TEC, Porto Rua do Campo Alegre, 687, 4169007 Porto, Portugal (e-mail: ofrazao@fc.up.pt).

Color versions of one or more of the figures in this paper are available online at http://ieeexplore.ieee.org.

Digital Object Identifier 10.1109/JLT.2013.2286223 of points to be analyzed along a structure reaches several hundred [1]. In these cases, distributed sensors should normally be preferred due to their low cost per monitored point, their simple arrangement and the geometric versatility of optical fibers. Distributed fiber sensors nowadays allow performing different types of measurements at any point along a fiber (strain, temperature, vibration, pressure, etc.). Among the distributed sensing techniques, optical time domain reflectometry (OTDR) was first demonstrated over three decades ago [2], [3] and is now a commonly used technique for distributed measurement of losses along the fiber (including the measurement of fiber attenuation, the location of broken points and optical connectors, discontinuities, etc.).

Traditional OTDRs use incoherent light and therefore can only measure intensity variations along the fiber. In a phasesensitive OTDR ( $\phi$ OTDR) however, a pulse of highly coherent light is injected into a conventional single-mode fiber and the light reflected from different scattering centers interferes coherently to produce the detected optical power trace [4]-[7]. Because the position of these scattering centers is random, the $\phi$ OTDR trace typically has random oscillations. This random pattern remains constant if the scattering centers do not suffer any change. However, in the case of localized vibrations, the trace shows variations synchronized with the vibration frequency. Due to its potential for fully distributed measurement of vibrations which can be used to monitor intrusions over large perimeters, $\phi \mathrm{OTDR}$ attracted considerable attention for more than two decades [8]. In fact, these sensors have been demonstrated in field tests, demonstrating enough sensitivity to detect people walking on top of a buried fiber [4]. In the literature, conventional $\phi$ OTDR systems have been shown to allow the distributed measurement of vibrations over dynamic ranges of a few tens of kilometers [5]. Distributed vibration measurements up to $8 \mathrm{kHz}$ with a resolution of $0.5 \mathrm{~m}$ were also demonstrated in [6]. In this case, a laser with a linewidth of $4 \mathrm{kHz}$, coherent detection and signal processing methods were required. Trustworthy vibration measurements can only be achieved with a good visibility $\left(V=\left(T_{\max }-T_{\min }\right) /\left(T_{\max }+T_{\min }\right)\right.$ where $T_{\max }$ and $T_{\min }$ are the maximum and minimum values of the trace) and high signal-to-noise ratio (SNR) in the trace. The increase of the input pump peak power will increase the dynamic range and SNR of a $\phi$ OTDR, but it is limited due to the onset of nonlinear effects [9]. As for the visibility of the trace, it will remain high if the coherence of the input laser is high (in comparison to the pulse width) and remains so all along the fiber. In temperature 
sensing, by analyzing the cross-correlation of $\phi$ OTDR traces with different input wavelength pulses, temperature and strain measurements have also been demonstrated [7].

In vibration-based structural damage identification or monitoring, ultrasonic waves are sometimes used to detect damages in the structure [10]. These waves can reach frequencies going from several $\mathrm{kHz}$ to $\mathrm{MHz}$. A distributed vibration sensor with capacity to detect these waves can therefore be required for these applications [11]. In this paper, the authors present an experimental and theoretical description of a high-visibility $\phi \mathrm{OTDR}$ and its performance when used for ultrasonic vibration measurements. The main novelty in the setup comes from the use of a semiconductor optical amplifier (SOA) to achieve high extinction ratio (ER) between pump pulses thus greatly decreasing the intra-band coherent noise of the setup. Due to spectral hole burning in the active material of the SOA, this component also increases the spectral purity of the laser in the active state. This combination allows performing frequency measurements in the limits set by the time of flight of the light pulses while maintaining the simplicity of the scheme, as no post-processing, extremely high coherence lasers or coherent detection are required. The optical intensity distribution in the $\phi$ OTDR trace is measured and the reflectance fading probability is calculated, showing good agreement with the theoretical model. The sensitivity of the vibration measurement is shown to increase with the average intensity of the trace in the measured point as predicted. The $\phi \mathrm{OTDR}$ trace was shown to have high visibility over a minimum of $9.9 \mathrm{~km}$. As for the vibration sensing, the optical power variations of the $\phi$ OTDR trace were shown to be synchronized with applied mechanical vibrations. The sensor was able to measure vibrations of up to $39.5 \mathrm{kHz}$, limited by the sampling frequency, with a resolution of $5 \mathrm{~m}$ in a demonstrated range of $600 \mathrm{~m}$ which could go up to $1.25 \mathrm{~km}$. If the range is reduced, the system could be used to read even higher frequencies.

\section{TheORETICAL MOdel OF A High-VISIBILITY $\phi$ OTDR}

\section{A. Signal Statistics}

We start by developing here a theoretical model for a highvisibility $\phi \mathrm{OTDR}$. This model will allow us to understand the performance of the experimental system described after. For the theoretical model, we assume that a short input pump pulse width $\left(\mathrm{w}_{p}\right)$ of highly coherent light is delivered into a fiber with constant loss $(\alpha)$ and isotropic propagation. This pulse reaches the position $z$ in the fiber at the time $t_{0} / 2$. The $\phi$ OTDR signal received from the pulse at that position (at the instant $t_{0}$ ) will be the sum of the fields reflected from the $M$ scattering points contained in section of the fiber $\mathrm{z} \in\left[\left(\mathrm{t}_{0} \mathrm{v}_{\mathrm{g}}-\mathrm{w}_{\mathrm{p}}\right) / 2,\left(\mathrm{t}_{0} \mathrm{v}_{\mathrm{g}}\right) / 2\right]$ and will be:

$$
E\left(t=t_{0}, z=0\right)=E_{0} e^{-2 \alpha \bar{z}} e^{i \omega t_{0}} \sum_{m=1}^{M} r_{m} e^{i \phi_{m}}
$$

where $V_{g}$ is the group velocity and $\bar{z}=\left[\mathrm{t}_{0} \mathrm{v}_{\mathrm{g}}-\left(\mathrm{w}_{p}\right) / 2\right] / 2$ the position corresponding to the pulse center from which the signal is being collected. The $m$ th scattering center has a reflectivity $r_{m} \in[0,1]$ (normally $\ll 1$ ) and the phase introduced in the reflected field by both the optical path and the scattering centers is $\phi_{m}$ (where $\phi_{m} \in[0,2 \pi]$ is assumed to be uniformly distributed). The amplitude of the reflected field will follow a Rayleigh distribution, as shown in previous papers [12]. The normalized intensity $(I)$ received at the extreme of the fiber, neglecting the losses, will therefore be [13]:

$$
\begin{aligned}
I & =|E|^{2}=\left|\sum_{m=1}^{M} r_{m} e^{i \phi_{m}}\right|^{2} \\
& =\sum_{m=1}^{M} r_{m}^{2}+2 \sum_{i=1}^{M-1} \sum_{j=i+1}^{M} r_{i} r_{j} \cos \left(\phi_{i}-\phi_{j}\right) .
\end{aligned}
$$

This addition of $M(M \rightarrow \infty)$ wave amplitudes is a random walk process and its probability distribution will follow an exponential distribution [13]:

$$
P\left(I_{M}\right)=\frac{1}{M} e^{\left(-\frac{I_{M}}{M}\right)} .
$$

It is interesting to note that the average intensity of the detected field from a given position will be proportional to the number of scattering centers $M\left(\left\langle I_{M}\right\rangle=\int_{0}^{+\infty} I_{M} P\left(I_{M}\right) d I_{M}=\right.$ $M)$ within the pulse length at that position.

As it can be seen there is a certain probability of having a close-to-zero signal at a given position (a signal below the noise level of the detector, $\delta$ ). This probability is termed "fading probability", since the system "loses" completely the signal at this position. The fading probability $\left(P\left(I_{M}<\delta\right)\right)$ as a function of the detector noise level $\delta$ will be the cumulative probability of the distribution function.

If a perturbation is introduced in the $q$ th $(q \in[1, M])$ scattering center with a corresponding phase perturbation of $\theta_{p}$, then the corresponding intensity variation $\left(I_{\Delta}\right)$ between the nonperturbed $(I)$ and the perturbed $\left(I^{\prime}\right)$ signals will be given by:

$$
\begin{aligned}
I_{\Delta} & =I-I^{\prime} \\
& =2 \sum_{i=1}^{q-1} \sum_{j=q}^{M} r_{i} r_{j}\left[\cos \left(\phi_{i}-\phi_{j}\right)-\cos \left(\phi_{i}-\phi_{j}-\theta_{p}\right)\right] .
\end{aligned}
$$

As it is visible, the response of the system turns out to be generally nonlinear, except for very small values of the perturbation $\theta_{p}$. The perturbed and unperturbed signals have correlated exponential probability distributions. Thus, the probability distribution of the intensity variation will be Laplacedistributed [13], [14] (a different form of exponential distribution). In the case of an applied vibration, a $\phi$ OTDR should therefore provide better information regarding the form (frequency) of the perturbation than its amplitude. Again, the signal variation is proportional to $M$ and the input signal intensity. A higher average back-reflected intensity $(I)$ will result in higher values of $I_{\Delta}$, increasing the sensitivity of the system and decreasing the fading probability of the signal of interest $\left(I_{\Delta}\right)$. The most obvious solution to reduce the fading probability is then to raise the pump power. However this approach is limited by the onset of optical nonlinearities [9]. 


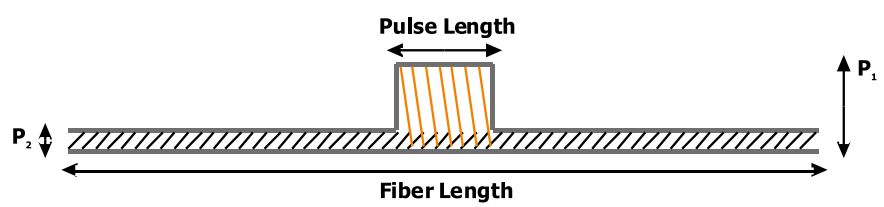

Fig. 1. Power distribution along the fiber for a $\phi$ OTDR input pump pulse with finite ER.

\section{B. Signal-to-Noise Ratio (SNR)}

As it has been seen previously, the minimum detectable signal will be limited by the noise in the optical detection. Two types of noise sources are present in a $\phi$ OTDR system: electrical noise caused by the photodetection process (including shot noise and thermal noise) and optical noise caused by fluctuations in the input power to the detector (introduced by optical amplifiers and coherent noise due to leakage of the pulse generation system). In high performance photodetectors like the ones available commercially in fiber-optic instrumentation, the noise contributions introduced by the photodetection process are close to the fundamental minimum. Considering usual parameters (pulse peak power in the order of hundreds of milliwatts, pulse length of a few meters, Rayleigh backscatter coefficient $\left(\alpha_{\text {Rayleigh }}\right)$ of $-70 \mathrm{~dB} / \mathrm{m}$ ), the SNR given by pure detection processes in a $100 \mathrm{MHz}$ detector is in the order of $20 \mathrm{~dB}$, using the traditional formula [15]. Optical noise is generally much more a problem as we will see next.

For a $\phi$ OTDR system like the one described in Section III, we can consider two main sources of optical noise: signalspontaneous and spontaneous-spontaneous beating caused by the backscattered amplified stimulated emission (ASE) from the optical amplifiers, and the non-infinite ER of the pulse generator. Fig. 1 presents the power distribution along the fiber for a $\phi$ OTDR input pump pulse with finite ER. Intra-band noise is generated due to the fact that there is optical power at the pump frequency outside the input pump pulse. The ratio between the peak power of the input pump pulse $\left(P_{1}\right)$ and the leaked power at the master frequency $\left(P_{2}\right)$ is the ER of the pulse generator used $\left(\mathrm{ER}=P_{1} / P_{2}\right)$. Considering an incoherent type of noise then: Intra-band SNR $\propto \mathrm{ER} * L_{\text {pulse }} / L_{\mathrm{fiber}}$, where $L_{\text {fiber }}$ and $L_{\text {pulse }}$ are respectively the lengths of the fiber and of the pulse in the fiber. However, if the coherence length of the master laser is much higher than the pulse and spreads over the complete fiber length (which is the case in most typical high-visibility $\phi$ OTDRs reported in the literature), then the backscattered continuous wave (cw) component will be interfering coherently with the backscattered pulse. In this case the detected power will have terms proportional to the input pump power $\left(\alpha_{\text {Rayleigh }} P_{1} L_{\text {pulse }}\right)$ (signal), the background $\left(\alpha_{\text {Rayleigh }} P_{2} L_{\text {fiber }}\right)$ (background noise) and the interference of the two $\left(\alpha_{\text {Rayleigh }} \sqrt{P_{1} L_{\text {pulse }} P_{2} L_{\text {fiber }}}\right)$ (coherent noise). Considering usual parameters $\left(P_{1} L_{\text {pulse }} \gg P_{2} L_{\text {fiber }}\right)$, the SNR will therefore be:

$$
\mathrm{SNR} \propto \sqrt{\operatorname{ER}\left(L_{\text {pulse }} / L_{\text {fiber }}\right)} .
$$

Putting typical figures, for $10 \mathrm{~km}$ ranges and $10 \mathrm{~m}$ pulse length, it is required an ER of $50 \mathrm{~dB}$ to achieve a SNR of the

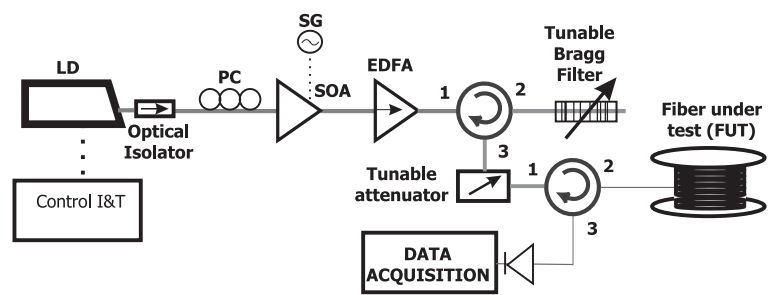

Fig. 2. Experimental setup: Acronyms are explained in the text.

order of $10 \mathrm{~dB}$. This is not easily attainable with conventional pulsing methods such as the electro-optic modulator. A high ER is therefore essential for reliable measurements. It is interesting to notice that an extremely high coherence laser will actually perform worse in a conventional system, as there is no improvement in the signal once the pulse coherence is higher than its length, but there will be an increase in the length over which coherence noise is introduced.

\section{EXPERIMENTAL SETUP AND CHARACTERIZATION OF THE HIGH-VISIBILITY $\phi$ OTDR}

The experimental setup used to characterize the highvisibility $\phi$ OTDR (shown in Fig. 2), is based on a minor adaptation of the setup presented in [9]. The input pump power was adjusted to achieve the best performance and avoid nonlinear effects (no spectral signature of MI was observed at the fiber end) [9]. A laser diode (LD) with a linewidth of $1.6 \mathrm{MHz}$ emitting at $1546 \mathrm{~nm}$ was used as the laser source. The used LD presents a high enough coherence for the experiment (LD coherence length $\approx 50 \mathrm{~m}$ and the pulse length in the fiber $\approx 10 \mathrm{~m}$ ) but not too high, thus minimizing the generation of coherent noise between pulses while maintaining the simplicity of the scheme (the LD is driven by a standard current and temperature controller) and not presenting the technological challenges typically presented by lasers with sub-MHz linewidths. An optical isolator was placed at the laser output to avoid disturbances in the laser coherence due to back-reflections. A SOA, with rise/fall times in the order $2.5 \mathrm{~ns}$, driven by a waveform signal generator was used to create $50 \mathrm{~ns}$ almost square pulses. A polarization controller was placed before the SOA to optimize the modulation properties and avoid any polarization sensitivity problem. Between the signal pulses, the SOA was negatively biased so as to enhance the ER of the delivered pulses. An ER of $>50 \mathrm{~dB}$ was achieved this way. In this configuration, the ER has a very high impact in the SNR of the detected trace. An erbium-doped fiber amplifier (EDFA) was used to boost the power of the input pulses and reach peak powers in the pulse of several hundred milliwatts. In order to minimize the effect of the ASE added by the EDFA, we inserted a tunable fiber Bragg grating (FBG) working in reflection. The spectral profile is the typical spectrum of a $100 \%$ reflection FBG and its spectral width is $0.8 \mathrm{~nm}$. For the used settings, the leaked ASE power is below the leaked power of the master signal and therefore the ER decrease is not significant after the EDFA + FBG [16]. Before being coupled into $9.9 \mathrm{~km}$ of SMF-28 fiber (FUT), light passed through an attenuator, which allowed varying the input power in 


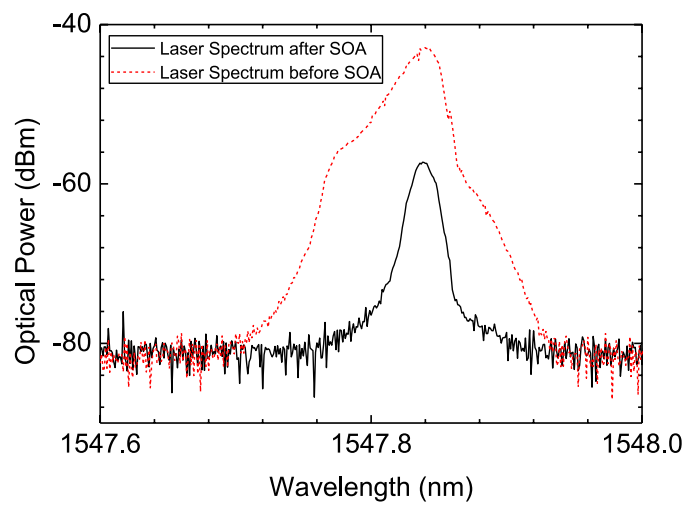

Fig. 3. Spectrum of a low coherence laser before and after passing through the SOA. Spectral hole burning in the SOA leads to a narrowband amplification of the central laser line, allowing a large reduction of side-mode noise.

the fiber. The average optical input power was measured at this position using a calibrated tap coupler (not shown in the figure) and the signal back-reflected from the fiber was recorded with a $125 \mathrm{MHz}$ p-i-n photo-detector and a high-speed digitizer. Since the $\phi$ OTDR traces exhibit high-contrast rapid oscillations, the detector bandwidth should ideally be much larger than the pulse spectral bandwidth. In our case the detector has a bandwidth (125 MHz) roughly six times higher than the pulse spectral bandwidth which is enough to adequately represent the process. In characterizing the visibility, we chose a long fiber in order to reliably ensure that the visibility conditions are kept in the final setup.

In this experiment the use of a SOA is very important due to the noise suppression between pulses which results in a much lower intra-band noise. Furthermore, due to spectral hole burning in the active material, the SOA enhances the spectral purity of the laser spectrum in the active state [17]. To evidence the impact of the SOA in the coherence of the launched spectrum, the laser was biased with a noisy current, leading to some spectral broadening of the central laser line. The spectrum of the laser was observed in an optical spectrum analyzer (OSA) before and after passing the SOA (see Fig. 3). The effect of the SOA is clearly visible as the spectrum after the SOA presents a much narrower linewidth (in fact limited by the resolution of the OSA of $0.02 \mathrm{~nm}$ ). In the conventional settings, the SOA helps to reduce noise introduced by the side modes of the laser, improving the trace noise.

The main picture of Fig. 4(a) shows a $\phi$ OTDR trace recorded with an input pump peak power of $\sim 400 \mathrm{~mW}$. In the inset figure the same trace is shown but the losses along the fiber have been numerically eliminated to improve the visualization. The trace displays the expected random oscillations around the average reflected power. The top figure shows the visibility of the $\phi$ OTDR interference signal along the fiber, calculated as indicated in the figure caption. Despite the fact that the amplitude of the oscillations and average reflected power decrease exponentially along the fiber due to the losses, the interference does not loose contrast along the propagation (the visibility remains close to 1). Since the sensitivity of the $\phi$ OTDR depends mostly on the visibility of the interferences, we can conclude that it re-

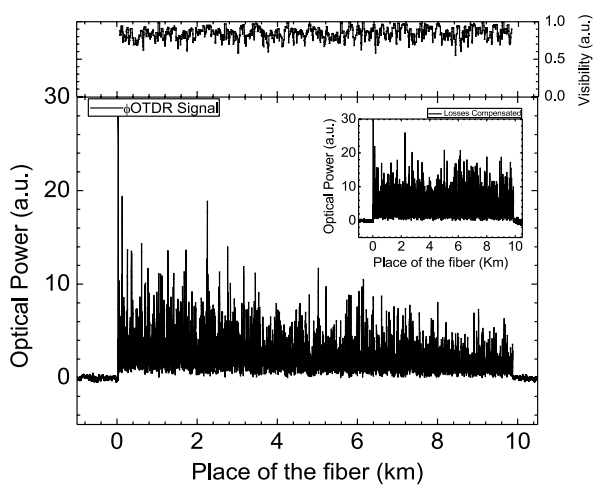

(a)

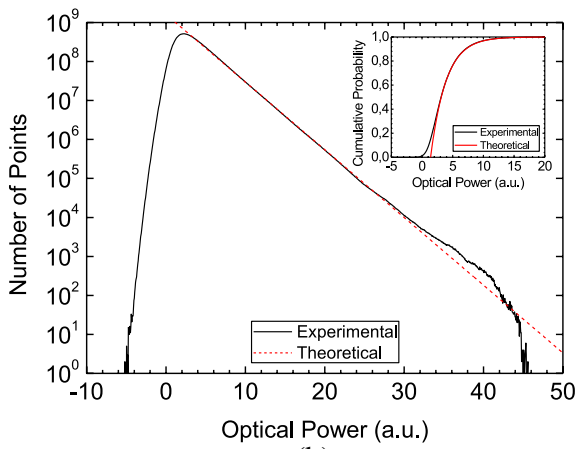

(b)

Fig. 4. (a) $\phi \mathrm{OTDR}$ interference signal along the FUT for an input pump peak power of $\sim 400 \mathrm{~mW}$ (inset figure: same $\phi$ OTDR signal where losses along the fiber have been eliminated to improve visualization). The top figure shows the visibility of the $\phi \mathrm{OTDR}$ interference signal. The visibility is computed as $V=\left(T_{\max }-T_{\min }\right) /\left(T_{\max }+T_{\min }\right)$ where $T_{\max }$ and $T_{\min }$ are the maximum and minimum values of the trace over a window of $40 \mathrm{~m}$. (b) Experimental $(5 \times$ $10^{6}$ traces obtained under the same conditions of the one presented in (a) over $\approx 10 \mathrm{~min}$ ) and theoretical optical intensity distribution in the $\phi \mathrm{OTDR}$ trace (inset figure: cumulative probability function).

mains high along the complete fiber length, although a normal decrease of sensitivity is expected toward the fiber end due to the pump pulse power attenuation along the fiber. The power limit (which ensures the maximum possible signal amplitude while avoiding nonlinear effects) obtained in this experiment will be later used also as the power limit for the shorter fiber. This way we ensure no visibility impairment due to modulation instability [9].

To characterize the intensity statistics and the fading probability in our setup, we obtained a histogram of the normalized intensities obtained from each position (fiber losses are numerically compensated) for $5 \times 10^{6}$ traces obtained under the same conditions of the one presented in Fig. 4(a) over $\approx 10 \mathrm{~min}$. The histogram is presented in Fig. 4(b). The beginning and end points of the fiber were excluded to avoid taking into account connector reflections and splice-induced reflections. The theoretical distribution curve (exponential) is also presented showing a good agreement with the experimental results. For low and high powers some discrepancies are observed which we consider acceptable given the bandwidth limitation of the detector and the perturbations induced by noise (they become relevant four orders of magnitude below the peak). The inset figure in Fig. 4(b) presents the cumulative probability function of the 
histogram, i.e., the reflectance fading probability for each optical power level. Again, a good agreement between the theory and the experimental results is obtained.

\section{VIBRATION MEASUREMENTS}

Vibration measurements are carried out by measuring consecutive traces and obtaining the power evolution as a function of time in each point along the fiber. The sampling frequency at each position corresponds therefore with the frequency at which the pump pulses are launched into the fiber, which is limited by the maximum length that can be inspected. As an example, to monitor $1 \mathrm{~km}$ of fiber requires that the pulse repetition rate is below $100 \mathrm{kHz}$ (each trace is $10 \mu \mathrm{s}$ long), which limits the maximum readable frequency at each position to $50 \mathrm{kHz}$. For the ultrasonic vibration measurements, the FUT was replaced with $600 \mathrm{~m}$ of SMF-28. Due to the limited re-trigger capability of our acquisition system, the pulse repetition rate could not be made larger than $80 \mathrm{kHz}$ and therefore the maximum readable frequency corresponds to $40 \mathrm{kHz}$ as given by Nyquist theorem. The far end of the fiber (around meter 590) was glued with tape inside a PVC tube $2 \mathrm{~m}$ long and $0.08 \mathrm{~m}$ of diameter in which mechanical vibrations of different frequencies were applied using a small vibration exciter with a maximum bare table acceleration of $736 \mathrm{~ms}^{-2}$ (with a $75 \mathrm{~g}$ mass attached). The fiber was clamped outside the tube in order to avoid the propagation of vibrations outside it. The optical power variation of the $\phi$ OTDR signal was monitored for the meter 590 (inside the tube) along consecutive traces. We started by using a $20 \mathrm{~Hz}$ vibration and monitoring with a sampling rate of $20 \mathrm{kHz}$ during $5 \mathrm{~s}$ with no post-processing. This gives us traces with very good SNR in the spectral domain, which allows us to characterize the statistical response of our system without being impaired by noise issues. The first $500 \mathrm{~ms}$ of the optical power variation are presented in Fig. 5(a). A clear pattern with peaks synchronized with the applied frequency is observed. The fast Fourier transform (FFT) of the optical power variation at the shaker location is presented in Fig. 5(b). A clear peak at $20.3 \mathrm{~Hz}$ appears, as well as smaller peaks in the second harmonic $(40.6 \mathrm{~Hz})$, and multiples of half of the fundamental frequency $(10 \mathrm{~Hz}, 30 \mathrm{~Hz})$. This nonlinear behavior is partly related to the non-linear response of the $\phi \mathrm{OTDR}$ itself and also to the mechanical response of the tube. Anyway, given the amplitude difference between the peaks, it can be envisaged that to some extent this nonlinearity can be corrected if the input stimulus is not too big. Considering the input pump pulse width $(50 \mathrm{~ns})$ the expected resolution should be of $5 \mathrm{~m}$. This is in agreement with our measurements since when the monitored position was more than $5 \mathrm{~m}$ away from the shaker position, the optical power variations and respective FFTs no longer showed sensitivity to the applied vibrations.

To characterize the sensitivity statistics of the $\phi$ OTDR, we varied the center frequency of the LD over a range covering $20 \mathrm{GHz}(160 \mathrm{pm})$. This was done by changing the bias current settings in the LD over a range of approximately $35 \mathrm{~mA}$ (with $\mu \mathrm{A}$ resolution). This allows us to record the vibration at the position of the shaker with varying settings in terms of average detected intensity (as the relative phases depend on the frequency of the

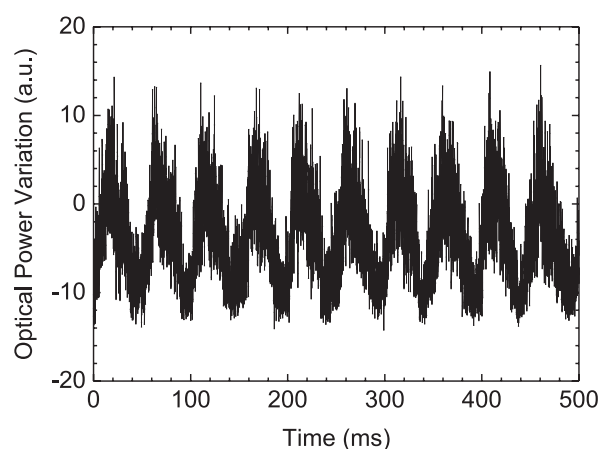

(a)

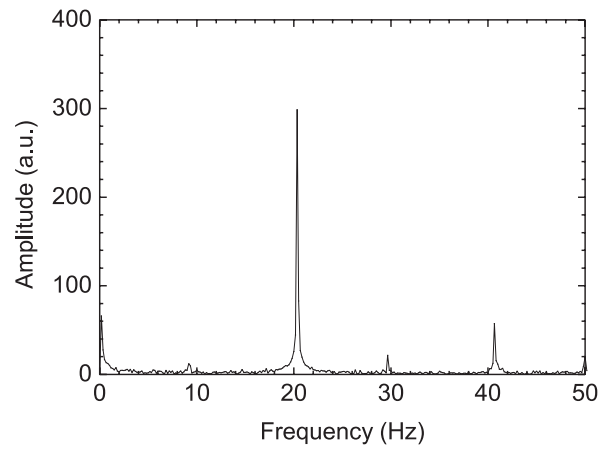

(b)

Fig. 5. (a) Optical power variation along time and (b) FFT of the optical power variation of the $\phi \mathrm{OTDR}$ signal in the position correspondent to the meter inside the tube $(590 \mathrm{~m})$ for an applied vibration of $20 \mathrm{~Hz}$.

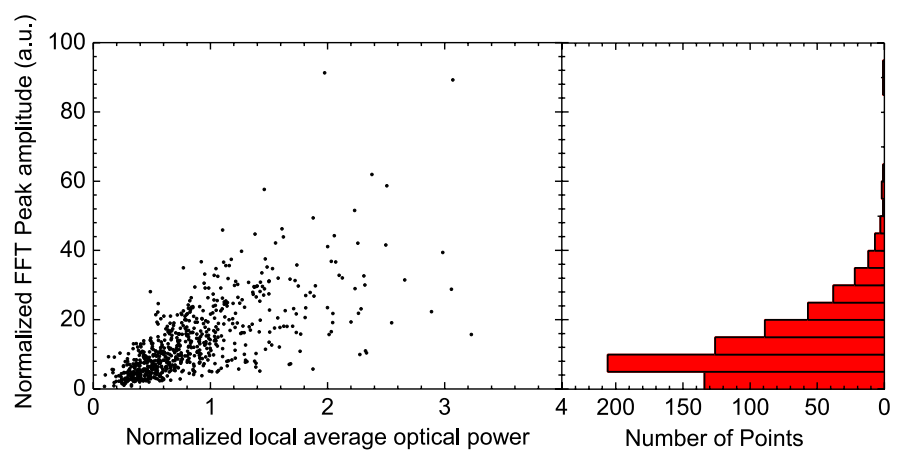

Fig. 6. Normalized sensitivity of the measurement as a function of the normalized average optical power of the $\phi \mathrm{OTDR}$ trace in the measured point (left) and its histogram (right).

laser, the interference in the shaker position experiences many maxima and minima over this sweep). For this entire sweep, we continuously recorded the amplitude of the applied $20 \mathrm{~Hz}$ mechanical vibration. Fig. 6 presents the normalized amplitude of the peak of $20 \mathrm{~Hz}$ in the FFT of the optical power variation in the shaker position as a function of the average optical power recorded in that meter. Both axis were divided by the average optical power recorded in order to compare equally all the points. The figure clearly shows that the sensitivity increases for higher average optical powers of the $\phi \mathrm{OTDR}$ trace in the measured point, in agreement with the theoretical model. In addition, the histogram shown on the right again displays the expected exponential behavior. 


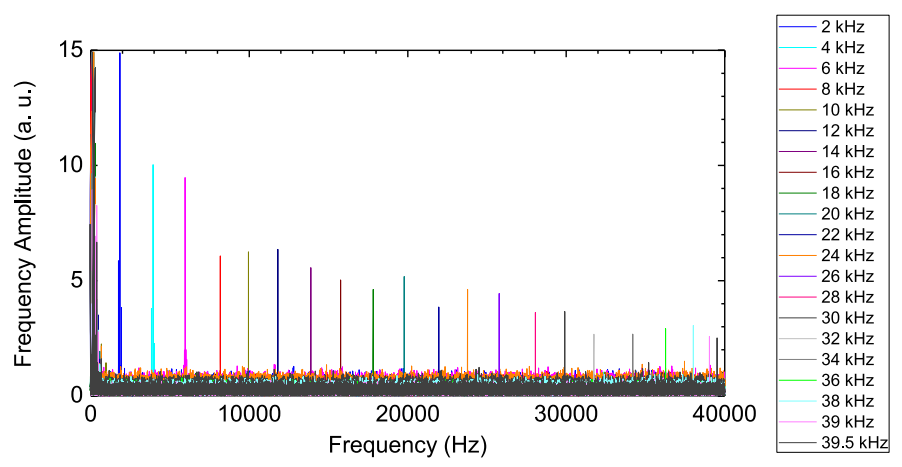

Fig. 7. FFT spectra of the optical power variation of the $\phi$ OTDR signal along time in a given meter for an applied vibration from 2 to $38 \mathrm{kHz}$ with intervals of $2 \mathrm{kHz}, 39 \mathrm{kHz}$ and $39.5 \mathrm{kHz}$.

In order to test the limits of acquisition of the system, the sampling rate was increased to $80 \mathrm{kHz}$ (maximum allowed due to the trigger holdoff of the acquisition system, $12 \mu \mathrm{s}$ ) and the acquisition time reduced to $1 \mathrm{~s}$. Fig. 7 presents the FFT spectra of the optical power variation recorded by the $\phi \mathrm{OTDR}$ at the position of the shaker $(590 \mathrm{~m})$ when the frequency applied to the tube is raised from 2 to $39.5 \mathrm{kHz}$. The recorded spectra showed clearly visible peaks in all the applied frequencies, with higher SNR and also higher non-linearity for lower applied frequencies. This corresponds to the fact that, as the frequency is reduced, the tube displacement is larger. For the maximum frequencies tested, the estimated displacement of the tube is in the sub-micrometer range, which illustrates clearly the extreme sensitivity of this technique. At low frequencies, multiples of $50 \mathrm{~Hz}$ are observed below $500 \mathrm{~Hz}$ which we believe could be owed to the mechanical actuator. Considering the clearly visible peak at $39.5 \mathrm{kHz}$, we believe that higher frequencies could still be recorded with higher sampling rates (not attainable with our acquisition system and this fiber length). Furthermore, although measurements were demonstrated in a fiber span of $600 \mathrm{~m}$, considering the previously demonstrated high visibility of the system in longer distances, no problem should be envisaged in extending the monitored fiber span to $1.25 \mathrm{~km}$ (limit allowed by the $80 \mathrm{kHz}$ sampling rate), if the trigger hold off of the acquisition system could be reduced to zero. It is worth mentioning that the presented spectra are not averaged or treated in any way, which means that the performance of the sensor could be increased with the proper data treatment for specific applications.

\section{CONCLUSION}

In this paper, the authors have presented an experimental and theoretical description of a high-visibility $\phi \mathrm{OTDR}$ and its performance when used for ultrasonic vibration measurements. The use of a SOA allowed signals with high SNR and the $\phi \mathrm{OTDR}$ trace was shown to have high visibility over the entire sensing range. The optical intensity distribution and the reflectance fading probability in the $\phi \mathrm{OTDR}$ trace was measured and found to be in good agreement with the theoretical model. The sensitivity of the vibration measurement is shown to increase with the average intensity of the trace in the measured point as predicted. An exponential distribution of the sensitivity was also found, again in good agreement with the theoretical expectation. As for the vibration sensing, the optical power variations of the $\phi \mathrm{OTDR}$ trace were shown to be synchronized with applied mechanical vibrations. Frequency measurements in the limits set by the time of flight of light were achieved while maintaining the simplicity of the scheme, as no post-processing, extremely high coherent lasers or coherent detection were required. The sensor was able to measure vibrations of up to $39.5 \mathrm{kHz}$, with a resolution of $5 \mathrm{~m}$ in a range which could go up to $1.25 \mathrm{~km}$.

\section{REFERENCES}

[1] X. Bao and L. Chen, "Recent progress in distributed fiber optic sensors," Sensors-Basel, vol. 12, no. 7, pp. 8601-8639, 2012.

[2] M. K. Barnoski, M. D. Rourke, S. M. Jensen, and R. T. Melville, "Optical time domain reflectometer," Appl. Opt., vol. 16, no. 9, pp. 2375-2379, 1977.

[3] M. K. Barnoski and S. M. Jensen, "Fiber waveguides - novel technique for investigating attenuation characteristics," Appl. Opt., vol. 15, no. 9, pp. 2112-2115, 1976.

[4] J. C. Juarez, E. W. Maier, K. N. Choi, and H. F. Taylor, "Distributed fiberoptic intrusion sensor system," J. Lightw. Technol., vol. 23, no. 6, pp. 20812087, 2005.

[5] J. C. Juarez and H. F. Taylor, "Field test of a distributed fiber-optic intrusion sensor system for long perimeters," Appl. Opt., vol. 46, no. 11, pp. 1968-1971, 2007.

[6] Z. Qin, L. Chen, and X. Bao, "Wavelet denoising method for improving detection performance of distributed vibration sensor," IEEE Photonic Tech Lett., vol. 24, no. 7, pp. 542-544, Apr. 2012.

[7] Y. Koyamada, M. Imahama, K. Kubota, and K. Hogari, "Fiber-Optic distributed strain and temperature sensing with very high measurand resolution over long range using coherent OTDR," J. Lightw. Technol., vol. 27, no. 9, pp. 1142-1146, 2009.

[8] H. F. Taylor and C. E. Lee, "Apparatus and method for fiber optic intrusion sensing," Texas A \& M University System, College Station, TX, USA, U.S. Patent 5194847, Mar. 16, 1993.

[9] H. F. Martins, S. M. Lopez, P. Corredera, P. Salgado, O. Frazão, and M. G. Herráez, "Modulation instability-induced fading in phase-sensitive optical time-domain reflectometry," Opt.Lett., vol. 38, no. 6, pp. 872-874, 2013.

[10] H. Cho and C. J. Lissenden, "Structural health monitoring of fatigue crack growth in plate structures with ultrasonic guided waves," Structural Health Monitoring, vol. 11, no. 4, pp. 393-404, 2012.

[11] Y. Botsev, E. Arad, M. Tur, I. Kressel, U. Ben-Simon, S. Gail, and D. Osmont, "Structural health monitoring using an embedded PZT-FBG ultrasonic sensor array," in Proc. 4th Eur. Workshop Structural Health Monitoring, Cracow, Poland, 2 Jul., 2008, pp. 649-656.

[12] P. Healey, "Fading in heterodyne otdr," Electron. Lett., vol. 20, no. 1, pp. 30-32, 1984.

[13] A. K. Wojcik, "Signal statistics of phase dependent optical time domain reflectometry," Ph.D. dissertation, Dept. Electr. Eng. Comput. Sci., Texas A\&M University, College Station, TX, USA 2006. Available: http://hdl.handle.net/1969.1/4873.

[14] S. Kotz, T. J. Kozubowski, and K. Podgórski, The Laplace Distribution and Generalizations: A Revisit With Applications to Communications, Economics, Engineering, and Finance. Boston, MA, USA: Birkhäuser, 2001, p. 23.

[15] G. P. Agrawal, Fiber-Optic Communication Systems, 3rd ed. New York, NY, USA: Wiley, 2002, pp. 156-158.

[16] D. M. Baney, P. Gallion, and R. S. Tucker, "Theory and measurement techniques for the noise figure of optical amplifiers," Opt. Fiber Technol., vol. 6, no. 2, pp. 122-154, 2000.

[17] J. Kim, M. Laemmlin, C. Meuer, D. Bimberg, and G. Eisenstein, "Static gain saturation model of quantum-dot semiconductor optical amplifiers," IEEE J. Quantum Electron., vol. 44, no. 7, pp. 658-666, Jul. 2008. 
Hugo F. Martins received the B.Sc. and M.Sc. degrees in physics from the University of Porto, Porto, Portugal, in 2009 and 2011, respectively. He is currently working toward the $\mathrm{Ph}$.D. degree under jointly-awarded $\mathrm{PhD}$ program in the University of Porto, Porto, Portugal and the University of Alcalá, Madrid, Spain.

During his M.Sc. dissertation work, he worked with INESC Porto in the development of fiber sensing applications using cooperative Rayleigh-Raman scattering. His current research interests include studying the possibility of using ultra-long fiber lasers based on distributed Rayleigh mirrors to obtain a new allfiber secure key distribution system (while working in Porto, with INESC TEC) and distributed fiber sensors, mainly the use of phase-sensitive optical time domain reflectometry for distributed vibration detection (while working in the University of Alcalá).

Sonia Martin-Lopez received the Ph.D. degree from the Universidad Complutense de Madrid, Madrid, Spain, in May 2006. The topic of her doctoral dissertation was on experimental and theoretical understanding of continuous-wave pumped supercontinuum generation in optical fibers. She had a Predoctoral stay in the Nanophotonics and Metrology Laboratory, Ecole Polytechnique Federale de Lausanne, Switzerland. She has been engaged as a Postdoctoral Researcher in the Applied Physics Institute and in the Optics Institute at the Spanish Council for Research during six years. Currently, she is a Postdoctoral Researcher in the Photonics Engineering Group at University of Alcalá, Madrid, Spain. She is author or co-author of over 100 papers in international refereed journals and conference contributions. Her current research interests include nonlinear fiber optics and distributed optical fiber sensors.

Pedro Corredera received the B.Sc. and Ph.D. degrees in physics from the University of Salamanca, Salamanca, Spain, in 1985 and 1989, respectively.

In 1989, he joined the Institute of Optics, CSIC, where he is currently working on IR radiometry and optical fibres metrology. From 1990-1991, he joined at National Physical Laboratory (NPL, Teddington /UK), working in Cryogenic Radiometry and optical fibre metrology. From 1996-2010, he joined the Institute of Applied Physics (IFA-CSIC) and he created a research group in Optical Communications Technologies (GiTCO) with three lines of work: optical fibers metrology, nonlinear properties of optical fibers and applications and optical fiber sensors. In 2010, he re-joined the Institute of Optics (IO-CSIC) in the group "nonlinear dynamics and fiber optics." He published more than 60 articles in scientific and technical journals and 100 contributions at international conferences. His current research interests include fiber-optic measurements, optical fiber sensors, nonlinear fiber optics, and IR radiometry and detection.

Dr. Corredera is a member of the Spanish Society of Optics, the Optical Society American and the European Optical Society.
Orlando Frazão received the Graduation degree in physics engineering (optoelectronics and electronics) from the University of Aveiro, Aveiro, Portugal, in 1999. He received the Ph.D. degree from the University of Porto, Porto, Portugal, in 2009, on optical fiber sensors based on interferometry and nonlinear effects.

From 1997 to 1998, he was with the Institute of Telecommunications, Aveiro, with a scholarship in the European Project UPGRADE (High Bitrate 1300-nm Upgrade of the European Standard Single Mode Network). Currently, he is a Senior Researcher and a head group of optical fiber sensor for Physical measurements at Optoelectronics and Electronic Systems Unit, INESC Porto. His current research interests include optical fiber sensors and optical communications. He has conducted several National research projects in optical sensing and optical communications and has also participated in an European project (NEXTGENPCF - Next Generation Photonic Crystal). He has several bilatera cooperation projects between France (Xlim, Université de Nice Sophie Antipolis), Spain (Universidad Carlos III de Madrid), Brazil [Universidade Federa do Para (UFPA)], and Poland (WRUT). He has published about 120 articles in peer-reviewed journals, over 220 papers in international and National conference proceedings, and authored 12 patents. He has been a reviewer for several international journals of the following societies: IEEE, OSA, Elsevier, IOP, and others. He has participated as part of the organizer committee of several conferences.

Dr. Frazão received two international Scientific awards. He is also a Senior Member of the SPIE.

Miguel González-Herráez received the M.Eng. and D.Eng. degrees from the Polytechnic University of Madrid, Madrid, Spain, in 2000 and 2004 respectively.

While working toward the D.Eng. degree, he worked first as a Research Assistant and then as Postdoctoral Fellow in the Applied Physics Institute at the Spanish Council for Research, Madrid, Spain, and had several long stays in the Nanophotonics and Metrology Laboratory, Ecole Polytechnique Federale de Lausanne, Switzerland. In October 2004, he was appointed Assistant Professor in the Department of Electronics, University of Alcalá, Madrid, Spain, where he was promoted to Associate Professor in June 2006. He is the author or coauthor of over 130 papers in international refereed journals and conference contributions and has given several invited talks at international conferences. His research interests include the wide field of nonlinear interactions in optical fibers.

Dr. González-Herráez has received several important recognitions to his research career, including the European Research Council Starting Grant and the "Agustin de Betancourt" prize of the Spanish Royal Academy of Engineering. He is also a Senior Member of the Optical Society of America.
Massimo L. Filograno received the "Laurea" (five year program) degree in electronic engineering from the Polytechnic of Bari, Bari, Italy, in 2009, discussing the thesis entitled "Real time monitoring of railway infrastructures using technology based on fiber Bragg grating". He has been working with the GRIFO (Group of Photonics Engineering of the University of Alcalá, Madrid, Spain) since September 2008, and with CSIC (the Spanish National Research Council) since September 2010, toward the Ph.D. degree of the Information Engineering Department, University of Alcala. His main research interests include optical fiber sensors applied to high speed train systems. 